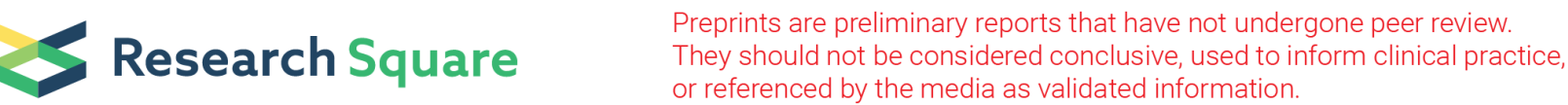

\section{Pregnancy-Related Ecological Shifts of Salivary Microbiota and its Association with Salivary Sex Hormones}

\section{Chenguang Niu}

Shanghai Jiao Tong University School of Medicine

Ting Dong

Shanghai Jiao Tong University School of Medicine

Wenxin Jiang

Shanghai Jiao Tong University School of Medicine

Li Gao

Shanghai Jiao Tong University School of Medicine

\section{Keyong Yuan}

Shanghai Jiao Tong University School of Medicine

Xuchen Hu

Shanghai Jiao Tong University School of Medicine

Wenzhen Lin

Shanghai Jiao Tong University School of Medicine

\section{Xuedong Zhou}

Sichuan University West China College of Stomatology

\section{Changen Xu}

Obstetrics and Gynecology Hospital of Fudan University

Zhengwei Huang ( $\sim$ huangzhengwei@shsmu.edu.cn )

Shanghai Jiao Tong University School of Medicine https://orcid.org/0000-0001-9861-514X

\section{Research article}

Keywords: Sex hormone levels, Salivary estradiol, Salivary progesterone, salivary microbiome, Miseq sequencing, Pregnancy

Posted Date: August 10th, 2020

DOI: https://doi.org/10.21203/rs.3.rs-54822/v1

License: (c) (i) This work is licensed under a Creative Commons Attribution 4.0 International License. Read Full License 


\section{Abstract}

Background: The interactions between hosts, oral microbiomes and microenvironments have been the subject of much research in recent years. Yet, whether the alterations in the host impact the oral microbiome is not understood well. The fluctuation of sex hormone levels during pregnancy is a dramatic change in the host, and is closely related to pregnancy-associated gingivitis. In this study, salivary estradiol and progesterone level were measured at three trimesters of pregnancy and after delivery ( $\mathrm{t} 1: \leq$ 14weeks $₫ \mathrm{t} 2$ : 20-25 weeks $₫ \mathrm{t}$ 3: 33-37 weeks; t4: 42 days after delivery) from 11 pregnant and 7 nonpregnant volunteers, and their salivary microbiome were collected and profiled by $16 \mathrm{~S}$ rDNA gene sequencing.

Results: The diversity of the salivary microbiome increased significantly in $\mathrm{t} 3$, compared to the $\mathrm{t} 1$ $(P<0.05)$, and a close parallel to the shift is found in the elevation of salivary sex hormones. Addionally, Capnocytophaga gingivalis, Peptoniphilus sp.oral taxon 386, Prevotella baroniae, Simonsiella muelleri and Lactobacillus reuteri were correlated to the fluctuation of sex hormone levels.

Conclusions: The diversity of the salivary microbiome in pregnant women was elevated with the gestation weeks, which was found parallel to the changes in estradiol and progesterone levels in saliva. Rather than highly abundant bacteria, the low abundant bacteria were more vulnerable to the host impact.

\section{Background}

The oral cavity has been considered to possess the second most complex microbiota in human body, only behind the colon. The host, the microbe and the microenvironment constitute the oral ecosystem. A widely accepted notion is that the interactions among hosts, oral microbiome and microenvironments play a vital role in protecting health[1-3]. Oral microbiota is dependent on the oral microenvironment and any change of the surroundings may cause the disturbance of oral microbiome.

A large shift was observed in the plaque bacterial communities in response to changes of the microenvironment in most oral and system diseases[4].For instance, an increase in radiation dose and damage in salivary glands of patients after head-and-neck radiotherapy directly influences the composition of plaque microbiota, consequently promoting oral diseases including dental caries and periodontitis[5]. Furthermore, oral microbiome is also confirmed to highly related to the hormonal changes. Serum levels of testosterone in boys and estradiol and progesterone in girls was detected to be positively correlated with levels of P. intermedia and P. nigrescens in a longitudinal study[6].

Pregnancy is a period in which the host undergoes a series of physiological changes, and among these, the dramatic fluctuation of sex hormone levels is highly significant. Meanwhile, pregnancy-associated oral diseases are commonly detected in the clinic and are reported to be closely related to the increase of sex hormones in saliva. Pregnancy-associated gingivitis is prevalent in about $50-70 \%$ of women during pregnancy[7]. In some cases, periodontitis is also reported to be more serious during pregnancy[8-10], 
which reflects the ecological imbalance of the oral microbiome during pregnancy. The relationship between sex hormones and separate oral pathogen has been investigated previously. Gursoy, M. et al. reported about increased growth of Prevotella negrescens during the second trimester among periodontitis-free women by using 16S rDNA-based PCR method[11]. Raber Durlacher et al. detected subgingival levels of Prevotella intermedia increased over 14 days of experimental gingivitis in pregnancy, but not post-partum[12]. DiGiulio DB et al. detected microbial samples collected weekly from the saliva in 49 pregnant women using 16S rRNA gene sequencing and found no significant change in alpha-diversity or beta-diversity across gestation[13].

Although much work has been done to explore the correlation of sex hormones and oral bacterial in different population[14], information about the impact of salivary sex hormone levels on the salivary microbiome throughout $16 \mathrm{~S}$ rDNA sequencing method is still limited. However, traditional methods are dependent on the ability of the bacteria to grow under artificial culture conditions, thereby limiting our knowledge of the overall microbiome. High-throughput sequencing methods provide an approach to explore the characteristic of bacterial communities at any classification level[15]. Therefore, the 16S rDNA sequencing method was used in this study for the purpose of exploring whether salivary sex hormones could be associated with the diversity and composition of bacterial communities in saliva, and providing a wider understanding of the interaction among the host, the microbe and the microenvironment.

\section{Results}

\section{Hormone levels in saliva during pregnancy and post- delivery}

Salivary progesterone and estradiol levels in pregnancy and postpartum are respectively shown in Fig. 1a and Fig. 1b, demonstrated by median (interquartile range). The concentrations of progesterone and estradiol had a similar trend. In the pregnant group, the salivary levels of both hormones increased during gestation ( $\mathrm{t} 1-\mathrm{t} 3)$ and reduced at post-delivery ( $\mathrm{t} 4)$. The peak was obtained at $\mathrm{t} 3$, the concentration of progesterone was $2255(1840,2981) \mathrm{pg} / \mathrm{ml}$; estradiol: $185.40(164.50 \otimes 204.20) \mathrm{pg} / \mathrm{ml}$. It was significantly higher than $\mathrm{t} 1$ (progesterone: $267.40(187.70 \otimes 286.00) \mathrm{pg} / \mathrm{ml}$; estradiol:17.23 (7.36ه23.43) pg/ml, $P<0.01$. After delivery (t4), the level of progesterone was 27.99 (16.69ه57.15) pg/ml; estradiol: 3.20 (2.54ه4.35) $\mathrm{pg} / \mathrm{ml})$. Compared with t3 and t2 which presented as progesterone: $619.32(589.20 \otimes 676.60) \mathrm{pg} / \mathrm{ml}$; estradiol: $31.01(21.60 \otimes 49.35) \mathrm{pg} / \mathrm{ml}$, value of both progesterone and estradiol of t4 were dramatically decreased $(P<0.05)$. Hormone levels in non-pregnant women had no significant change from the first visit (t1) to the fourth visit (t4).

\section{Salivary microbiota in pregnant and non-pregnant women}

Among 3,233,576 valid sequences obtained from 71 salivary samples, 2,928,959 reads passed the quality test, with an average of 41,253 reads per sample. After alignment in the Greengenes database, the representative sequences were classified into 598 OTUs at the $97 \%$ cut level. 
In this study, the core microbiome was defined as a co-occurrence bacterial community that could be deteced at every time point in each individual. Sixteen phyla could be detected from 71 samples (Fig. 2a). The phylum core which represented $99.46 \%$ of the total sequences were composed of Firmicutes, Proteobacteria, Bacteroidetes, Fusobacteria, Actinobacteria and Spirochaetes (Fig. 2b). Among the six phyla, Firmicutes accounting for $32.71 \%$ and $36.20 \%$ of pregnant and non-pregnant women, respectively, was the most abundant taxa, followed by Proteobacteria (28.27\% for the pregnant group and $28.63 \%$ for the non-pregnant group), Bacteroidetes (24.18\% for the pregnant group and $22.33 \%$ for the non-pregnant group). However, no significant differences could be calculated from the relative abundance of core phyla in two groups, meaning that phyla core was a stable bacterial community despite the alterations in gestation.

At the genus level, 173 genera were detected from all samples. Among them, 172 taxa could be found in the oral habitat of pregnant women, and 161 taxa were in the non-pregnant group. The most enriched 50 genera for all samples were demonstrated by Heatmap (Fig. 2c). Within 173 genera, 31 taxa comprised the genera core community, which represented $95.09 \%$ of all reads (Fig. 2d). Notable differentiations were presented in the relative abundance of Haemophilus, Veillonella, Gemella, which were enriched in nonpregnant women $(P<0.05)$. Furthermore, results of PCA (Principal Components Analysis)based on the genus level classification indicated no clear segregations in community structures among pregnant groups ( $\mathrm{t} 1, \mathrm{t} 2, \mathrm{t} 3, \mathrm{t} 4)$, with the first 2 principal components representing $38.74 \%$ and $20.67 \%$ of the total variations (Fig. S1).

Diversity of the salivary microbiome in the pregnant group was higher than that in non-pregnant women

The diversity of salivary bacterial communities in the pregnant and non-pregnant groups was evaluated by the Shannon diversity index (Fig. 3). In the pregnant group, the diversity of the bacterial community was closely related to the weeks of gestation, as revealed by the Shannon index. From early to late pregnancy ( $\mathrm{t} 1-\mathrm{t} 3)$, a gradually increasing Shannon diversity index was obtained. After delivery ( $\mathrm{t} 4)$, the diversity of the salivary microbiota declined. At the third trimester, the diversity of the salivary microbiota in pregnant women was significantly higher than that in $\mathrm{t} 1(P<0.05)$.

\section{Composition differences in microbiota between pregnant and non-pregnant women}

In addition to the characteristic core bacterial communities, several other taxa were also present, and their relative abundance in the two groups was analyzed. To identify these variable taxa, linear discriminate analysis coupled with size effect (LEfSe) measurements was applied to the data. As expressed by a circular cladogram in Fig. 4a, taxa with significant differences in relative abundance between the two groups at each cut-off level were observed. The only phylm that had a different relative abundance in the two groups was Thermi; it was enriched in the non-pregnant group. At the genus level, among the 20 genera, 14 genera were more abundant in the pregnant group. Determined by LDA score in Fig. 4b, Cardiobacterium was discovered to be the most discriminative genera in pregnant women within the 14 


\section{The correlation of salivary sex hormone levels to the salivary bacterial communities}

To explore the genus-level phylotypes of the salivary bacterial communities associated with salivary sex hormone levels, an RDA based approach was used in two steps[16]. Step one : distinguishing the pregnancy associated OTUs; Step two: testing these OTUs for their responses to the salivary sex hormone gradient responses. At the first step of RDA (Fig. 5a), pregnancy and non-pregnancy were considered as the environmental variables, and the relative abundance of OTUs of all samples was used as the species variable. The Monte Carlo Permutation Procedure (MCPP) showed that the constrained ordination model by the non-pregnant or pregnant group was significant $(P=0.012)$.From the first step of RDA, the samples composition shifts had shared $2.02 \%$ variability of the salivary microbiota. In Fig. 5 a, each blue arrow represented an OTU. Arrows with acute angles or obtuse angles with the treatment centroids indicated a positve or negative correlation respetively. Totally 223 OTUs (both positive and negative) were detected to be associated with pregnancy. Then, in the second step of RDA (Fig. 5b), the salivary sex hormone was considered as the environmental variable, and the 223 OTUs selected from the first step of RDA (Fig. 5a) become the species variables. The MCPP showed that the model was also significant $(P=0.042)$. Eventually, 63 OTUs had shared $1.68 \%$ variability of the salivary microbiome. Among these 63 OTUs, the relative abundance of 43 OTUs (blue arrows with the smaller angle with the environmental variable) was analyzed with respect to the elevation of salivary sex hormones levels. All 63 OTUs are shown in Fig. 5c. Forty-three OTUs were enriched in the pregnant group and the other 20 OTUs were more abundant in the non-pregnant group.

Pearson correlation coefficient and regression analysis were used to analyze the correlation of sex hormone levels and salivary bacteria. Five taxa (Capnocytophaga gingivalis, Peptoniphilus sp.oral taxon 386, Prevotella baroniae, Simonsiella muelleri and Lactobacillus reuteri) were detected to be significantly relevant to the fluctuation of sex hormone during pregnancy and after delivery (Fig. 6). The regression analysis presented as Fig. S2.

\section{Discussion}

Saliva is a microenvironment for microbiota that acts as a bridge for the host and microbe to communicate with each other. Any disturbance of the microenvironment may contribute to the alteration of oral microbe and its relationship with host. Dramatic fluctuations of salivary hormone levels were observed in this study, which were similar to previous reports on pregnant and non-pregnant women. Figuero et al. measured the concentration of progesterone and estradiol in saliva with a similar longitudinal design (duration of pregnancy and 3-months postpartum), and found that hormone levels increased greatly during pregnancy, peaked at the third trimester and dropped after delivery[17]. In another study, Khosravisamani et al. evaluated the effect of the menstrual cycle on salivary progesterone 
and estradiol levels in non-pregnant women and found that values determined during ovulation (on days 12-14) and the pre-menstruation trimesters (on days 22-24) were significantly higher than at other days[18]. Therefore, in this study, the sampling time selected for non-pregnant women was days 17-21 within the menstrual cycle, and the results obtained were in agreement with previous findings.

The correlation between pregnancy and the oral bacteria has been profiled in many reports. On the one hand, accumulating evidence has indicated that oral bacteria play a significant role in intrauterine infections, which is a major cause of complications of pregnancy, including preterm birth and still birth $[19,20]$. One the other hand, both clinical and laboratory studies have shown that oral diseases such as gingivitis and periodontitis are likely to worsen during pregnancy. The increase in sex hormone levels in saliva might be responsible for this, and the shift in dominant pathogens during pregnancy and its correlation with sex hormones has been demonstrated previously[20, 21].

It is worth noting that dramatic fluctuation of hormone levels might have a potential impact on the diversity of the salivary microbiome. This was evident in the results that the Shannon index of the salivary microbiome increased with the level of sex hormones. Both the highest concentration of salivary sex hormones and the richest Shannon index were found at the third trimester ( $\mathrm{t} 3,33-37$ weeks), and these values were reduced significantly after delivery. A higher Shannon index represents a higher diversity for the bacterial community. A similar trend in the Shannon index was obtained during pregnancy and after delivery, suggesting that the diversity of salivary microbial communities might be associated with the fluctuation of sex hormone levels detected in saliva. A possible explanation for the diversity between two groups is that the sex hormone levels promoted the colonization of different oral species[14]. The sex hormones could serve as a nutrient for some bacteria. Kornman et al. have demonstrated that pigmented Prevotella intermedia sensulato and Prevotella melaninogenica are able to substitute progesterone and estradiol for vitamin $\mathrm{K}$, which is needed as an essential factor for their growth[21].

A co-occurrence bacterial community was observed and consisted of the core microbiome in this study. There is substantial evidence that the core microbiome is likely to be a group of bacteria that is stable over time and represents high relative abundance[22, 23]. Findings of the present study are consistent with this observation. From the level of the phylum to that of the genus, the core microbiome represents $99.46 \%$ and $95.09 \%$, respectively, of relative abundance in pregnant women. No obvious composition differences could be found in the core microbiome at the phylum level between the two groups. However, more differentiation of low relative abundance taxa between pregnant and non-pregnant women were observed throughout the Lefse and LDA scores. As revealed by the Lefse and LDA scores, fourteen genera that were found to be significantly abundant in pregnant women represented a low relative abundance, and only three of six genera that were enriched in non-pregnant women were from the core microbiome. This interesting observation implies that it is the low abundance bacteria that mainly comprise the community of differential taxa between the pregnant and non-pregnant women. The importance of bacteria with low relative abundance has been widely reported in many fields, and might play an 
important role in the occurrence and process of diseases[24, 25]. Therefore, bacteria with low relative abundance should not be ignored.

To explore all the relevant bacteria that are associated with the fluctuation of salivary sex hormones during pregnancy, RDA analysis was applied in this study. Forty-three OTUs were observed to be related to the increase in the sex hormone levels, and the other 20 OTUs were negatively correlated with the hormones. This indicates that, among the 63 OTUs detected to be related to pregnancy by the bioinformatics strategy, more than two-thirds of the OTUs in saliva are enriched by the sex hormones. A possible explanation is that the increase in the sex hormones may influence directly the periodontal tissues[26, 27]. For instance, estradiol is reported to be a considerable factor in the proliferation, regeneration and differentiation of gingival epithelial cells and fibroblasts, and subsequently causes a reduced epithelial barrier to invasive bacteria[28]. Moreover, among the 63 hormone-related OTUs, 10 included Prevotella spp. The correlation of Prevotella spp. and pre'gnancy has been widely reported[14, 29]. Dareen Fteita and his colleague have reported that Prevotella nigrescens, Prevotella pallens and Prevotella aurantiaca, which are similar to Prevotella intermedia, have a higher relative abundance as estradiol levels increase during pregnancy[30].

Lactobacillus reuteri, Capnocytophaga gingivalis and Prevotella baroniae were detected by Pearson correlation coefficient to be relevant with the sex hormone levels in pregnant women. Lactobacillus also had higher LDA scores in pregnant group. It implies that Lactobacillus is significantly elevating in pregnant group and sex hormones might play a role in it. Lactobacillus reuteri is often used in the probiotic therapy to prevent and manage the oral inflammatory diseases through immunomodulation and down regulation of the inflammatory cascade. It was reported that Lactobacillus reuteri is useful in treating gingivitis in diabetic patients[31]. Capnocytophaga gingivalis was a periodontopathogenic bacteria. Emerging evidence detected the relationship between Capnocytophaga gingivalis and chronic periodontitis[32]. Santa Cruz I et al. also found that the presence of Capnocytophaga spp. were significantly associated with low-birth weight[33]. Therefore, it was suggested from this study that Lactobacillus reuteri and Capnocytophaga gingivalis might have antagonism effect. Prevotella baronia was found to be a relative of Prevotella intermedia[34]. Prevotella intermedia is a well-known periodontal pathogen and contributes a lot to the progress of pregnancy related gingivitis and periodontitis. Prevotella baronia is also reported to be found in primary endodontic infections and acute apical abscesses[35, 36]. However, direct evidence between sex hormones and Prevotella baronia need further investigations.

In this study, it is unable to avoid that small sample size and other potential factors such as diet habits, work, BMI and vitamin usage may have an impact on the results. Since it is an exploratory analysis, preliminary data were provided to suggest the correlation of salivary microbiome and salivary sex hormones, experimental procedures need to be done to explore the exact evidence between sex hormones and related microbes.

\section{Conclusions}


The main conclusion of this study is that the diversity of the salivary microbiome in pregnant women elevated with the gestation weeks, which is parallel to the alteration in estradiol and progesterone levels in saliva. Low abundant taxa have a relative larger fluctuation than the high abundant bacteria during pregnancy. Overall, 63 OTUs are closely related to the levels of salivary sex hormones and Prevotella spp. are the predominant species that are affected.

\section{Methods}

\section{Study design and subject recruitment}

This study followed the Declaration of Helsinki on medical protocol and ethics and obtained the approval from the regional Ethical Review Board of Shanghai 9th Hospital affiliated to Shanghai Jiaotong University, School of Medicine. The written informed consent was obtained from all subjects. Pregnant subjects were recruited from the Shanghai Red House Obstetrics and Gynecology Hospital, and nonpregnant volunteers were from Ninth people's Hospital, Shanghai Jiao Tong University School of Medicine. In the initial recruitment, eleven pregnant subjects who were in the first trimester (average gestation age: $13.90 \pm 2.5$ weeks) enrolled in this study, and were sampled four times: the first trimester (t1: $\leq 14$ weeks), the second trimester (t2: 20-25 weeks), the third trimester (t3: 33-37 weeks) and postpartum (t4: 42 days after delivery). Seven healthy non-pregnant women enrolled in the study, and all of them complied with the four visits at two-month intervals visit (t1-t4) (admission criteria are shown in Table S1). Saliva samples of all subjects were collected at four time points ( $\mathrm{t} 1-\mathrm{t} 4)$. The general description of subjects was demonstrated in Table S2.

\section{Saliva collection}

For each participant, unstimulated saliva was collected at least 1 hour after eating. Subjects were asked to rinse their mouths with water, wait for 2 minutes, and then use a disposable sterile syringe (with the tip removed) to suction off $1.5 \mathrm{ml}$ saliva into two sterile EP tubes $(2 \mathrm{ml})$. For each individual, one tube $(1.5 \mathrm{ml})$ was used for extracting DNA, and the other $(1.5 \mathrm{ml})$ for hormone level determination. All specimens were transported on ice immediately to the laboratory, and then stored at $-20^{\circ} \mathrm{C}$ until the determination of hormone levels and extraction of DNA.

\section{Progesterone and estradiol concentration analysis}

The saliva samples were centrifuged at $1077 \mathrm{rpm}$ for $5 \mathrm{~min}$; then, the supernatants were collected and analyzed. Salivary estradiol and progesterone concentrations were measured using a kit obtained from Demeditec (Kiel Wellsee, Schleswig-Holstein, Germany). The lowest detectable level of estradiol that can be distinguished from the zero standard is $0.4 \mathrm{pg} / \mathrm{mL}$. The lowest detectable level of progesterone that can be distinguished from the zero calibrator is $5.0 \mathrm{pg} / \mathrm{mL}$.

\section{DNA extraction and 16S rDNA sequencing}


Genomic DNA was isolated using the standard protocol of a bacterial genomic DNA extraction kit (QIAGEN, Valencia, CA,USA) in a sterile environment. DNA was quantified by NanoDrop and PicoGreen assays[16]. All extracted DNA was stored at $-80^{\circ} \mathrm{C}$. A unique 8-base "barcode" was attached to the $5^{\prime}$ end for each sample and the polymerase chain reaction (PCR) amplification of the 16S rDNA hypervariable V4-V5 region was carried out using the forward primer 515 (F, forward primer: 5'-GTG CCA GCM GCC GCG GTA A-3'), 926 (Rロreverse primer: 5'-CCG TCA ATT YYT TTR AGT TT-3'). The barcodes of all samples were showed in Table S3. The amplicon sequencing was performed with the lllumina Miseq platform (PE 300) according to a standard protocol. The sequences which $<150 \mathrm{bp}$, had average Phred scores of $<25$, contained ambiguous bases, or were confirmed as chimeric artifacts were discarded. Uchime in MOTHUR (1.31.2) was used to get rid of the chimera sequences. The qualified sequences were submitted to the SILVA database (SILVA 106; http://www.arb-silva.de) for taxonomic analysis.

\section{Bioinformatic analysis and statistical analysis}

MOTHUR (http://www.mothur.org/wiki/Shannon) was applied to generate the operational taxonomic units (OTUs) and OTU rarefaction curves. The Shannon diversity index and the taxa abundances at the phylum, genus and species levels were also calculated by QIIME (version 1.8.0) at $97 \%$ identity. Statistical analysis was conducted using software GraphPad Prism version 6.01 and SPSS Statistics 22.0. After normality (Kolmogorov-Smirnov test) and homogeneity of variance (Levene's test) were tested, KruskalWallis test and Dunn's multiple comparisons test were used to analyze significant differences in Shannon index and salivary progesterone and estradiol level[37]. The results presented by column diagram (median with interquartile range).

LEfSe (version 1.0) was used to find differentially abundant genera in the pregnant and non-pregnant group for biomarker discovery using the online Galaxy workflow framework

(http://huttenhower.sph.harvard.edu/galaxy/). For the first step, Kruskal-Wallis (KW) sum-rank test was used to detect the biomarker among classes (Alpha value, $P<0.05$ ). In the second step, pairwise Wilcoxon test was performed to do the comparisons among the sub-classes (Alpha value, $P<0.05$ ). In the last step, the linear discriminant analysis (LDA) score demonstrated the magnitude of the distinct relative abundance between the two groups. The LDA score for discriminative features was set to 2.0. The bigger LDA score related to a larger difference.

Redundancy analysis (RDA) was performed using CANOCO for Windows 4.5 (Microcomputer Power, USA). The result of DCA analysis suggested the length of gradient $<3$, therefore, RDA rather than CCA analysis was used here.

Pearson correlation coefficient is a direct gradient analysis technique used to analyze the linear relationships between components of response variables with a set of explanatory variables. Before started, Shapiro-wilk test was used to assess the normality. FDR (false discovery rate) correction was used to correct the $P$-value in multiple hypothesis test. 


\section{Abbreviations}

PCR

polymerase chain reaction

OTUs

operational taxonomic units

LDA

linear discriminant analysis

LEfSe

linear discriminate analysis coupled with size effect

\section{Declarations}

\section{Ethics approval and consent to participate}

This study was approved by the Ethical Committee of Shanghai Ninth people's Hospital, Shanghai Jiao Tong University School of Medicine, China. Informed written consent was obtained from all subjects. Pregnant subjects were recruited from the Shanghai Red House Obstetrics and Gynecology Hospital, and non-pregnant volunteers were from Shanghai Ninth Hospital.

\section{Consent for publication}

Not applicable

\section{Availability of data and materials}

All the raw sequences were deposited in the NCBI Sequence Read Archive database. The accession number is SRP113577

\section{Competing interests}

The authors declare that they have no competing interests.

\section{Funding}

This work was supported by a grant from the National Natural Science Foundation of China (NSFC) (No. 81570964/81371143区61370131); Chinese Academy of Sciences (No. XDA01030502), Science and Technology Commission of Shanghai Municipality (No. 14431900900/16430723500/17140903500) and was partly supported by Shanghai Summit \& Plateau Disciplines.

\section{Authors' contributions}

LCG drafted the main manuscript. DT contributed to drafting and revising the manuscript. JWX analyzed and interpreted the data. GL, YKY, HXC and LWZ helped to the samples collection and data analysis. ZXD 
contributed to the design of the study. XCG and HZW designed the main scheme of the research and modified the manuscript. All authors read and approved the final manuscript.

\section{Acknowledgements}

We express our sincere appreciation to all the volunteers who took part in this study. We also acknowledge Shanghai Personal Biotechnology Co., Ltd. for their kind help in Miseq sequencing and bioinformatics analysis.

\section{References}

1. Aw V. Discuss the role of microorganisms in the aetiology and pathogenesis of periapical disease. Aust Endod J. 2016;42(2):53-9.

2. Gholizadeh $P$, et al. Role of oral microbiome on oral cancers, a review. Biomed Pharmacother. 2016;84:552-8.

3. Uriarte SM, Edmisson JS, Jimenez-Flores E. Human neutrophils and oral microbiota: a constant tugof-war between a harmonious and a discordant coexistence. Immunol Rev. 2016;273(1):282-98.

4. Gao L, et al. Oral microbiomes: more and more importance in oral cavity and whole body. Protein Cell. 2018;9(5):488-500.

5. Hu YJ, et al. Exploring the dynamic core microbiome of plaque microbiota during head-and-neck radiotherapy using pyrosequencing. PLoS One. 2013;8(2):e56343.

6. Nakagawa $S$, et al. A longitudinal study from prepuberty to puberty of gingivitis. Correlation between the occurrence of Prevotella intermedia and sex hormones. J Clin Periodontol. 1994;21(10):658-65.

7. Han YW. Can oral bacteria cause pregnancy complications? Womens Health (Lond). 2011;7(4):4014.

8. Srinivas SK, Parry S. Periodontal disease and pregnancy outcomes: time to move on? J Womens Health (Larchmt). 2012;21(2):121-5.

9. Carrillo-de-Albornoz A, et al. Gingival changes during pregnancy: II. Influence of hormonal variations on the subgingival biofilm. J Clin Periodontol. 2010;37(3):230-40.

10. Toygar HU, et al. Periodontal health and adverse pregnancy outcome in 3,576 Turkish women. $J$ Periodontol. 2007;78(11):2081-94.

11. Gursoy $M$, et al. Does the frequency of Prevotella intermedia increase during pregnancy? Oral Microbiol Immunol. 2009;24(4):299-303.

12. Raber-Durlacher JE, et al. Experimental gingivitis during pregnancy and post-partum: clinical, endocrinological, and microbiological aspects. J Clin Periodontol. 1994;21(8):549-58.

13. DiGiulio DB, et al., Temporal and spatial variation of the human microbiota during pregnancy. 2015. 112(35): p. 11060-5. 
14. Kumar PS, Sex and the subgingival microbiome: do female sex steroids affect periodontal bacteria? Periodontol 2000, 2013. 61(1): p. 103 - 24.

15. Loman NJ, et al. Performance comparison of benchtop high-throughput sequencing platforms. Nat Biotechnol. 2012;30(5):434-9.

16. Chen $\mathrm{H}$, et al. A Filifactor alocis-centered co-occurrence group associates with periodontitis across different oral habitats. Sci Rep. 2015;5:9053.

17. Figuero E, et al. Gingival changes during pregnancy: I. Influence of hormonal variations on clinical and immunological parameters. J Clin Periodontol. 2010;37(3):220-9.

18. Khosravisamani $M$, et al. Effect of the menstrual cycle on inflammatory cytokines in the periodontium. J Periodontal Res. 2014;49(6):770-6.

19. Siqueira FM, et al. Intrauterine growth restriction, low birth weight, and preterm birth: adverse pregnancy outcomes and their association with maternal periodontitis. J Periodontol. 2007;78(12):2266-76.

20. Chatterton RT Jr, et al. Characteristics of salivary profiles of oestradiol and progesterone in premenopausal women. J Endocrinol. 2005;186(1):77-84.

21. Kornman KS, Loesche WJ. Effects of estradiol and progesterone on Bacteroides melaninogenicus and Bacteroides gingivalis. Infect Immun. 1982;35(1):256-63.

22. Zaura E, ten Cate JM. Towards understanding oral health. Caries Res. 2015;49(Suppl 1):55-61.

23. Huse SM, et al. A core human microbiome as viewed through $16 \mathrm{~S}$ rRNA sequence clusters. PLoS One. 2012;7(6):e34242.

24. Campbell AG, et al. Diversity and genomic insights into the uncultured Chloroflexi from the human microbiota. Environ Microbiol. 2014;16(9):2635-43.

25. Schulze-Schweifing K, Banerjee A, Wade WG. Comparison of bacterial culture and 16S rRNA community profiling by clonal analysis and pyrosequencing for the characterization of the dentine caries-associated microbiome. Front Cell Infect Microbiol. 2014;4:164.

26. Wu M, Chen SW, Jiang SY, Relationship between gingival inflammation and pregnancy. Mediators Inflamm, 2015. 2015: p. 623427.

27. Yuan G, et al. Progesterone modulates the proliferation and differentiation of human periodontal ligament cells. Calcif Tissue Int. 2010;87(2):158-67.

28. Gursoy M, et al. High salivary estrogen and risk of developing pregnancy gingivitis. J Periodontol. 2013;84(9):1281-9.

29. Machado FC, et al. Longitudinal study on clinical and microbial analysis of periodontal status in pregnancy. Braz Oral Res. 2016;30(1):e87.

30. Fteita D, et al. Does estradiol have an impact on the dipeptidyl peptidase IV enzyme activity of the Prevotella intermedia group bacteria? Anaerobe. 2015;36:14-8.

31. Sabatini S, et al. Oral probiotics in the management of gingivitis in diabetic patients: a double blinded randomized controlled study. J Biol Regul Homeost Agents. 2017;31(2 Suppl 1):197-202. 
32. Camelo-Castillo A, et al. Relationship between periodontitis-associated subgingival microbiota and clinical inflammation by 16S pyrosequencing. J Clin Periodontol. 2015;42(12):1074-82.

33. Santa Cruz l, et al. Association between periodontal status and pre-term and/or low-birth weight in Spain: clinical and microbiological parameters. J Periodontal Res. 2013;48(4):443-51.

34. Petrukhina NB, et al. [Study of mutual dependence of periodontal and colonic microbiome in health and pathology using NSG-sequencing]. Stomatologiia (Mosk). 2016;95(2):8-13.

35. Rocas IN, Siqueira JF Jr. Prevalence of new candidate pathogens Prevotella baroniae, Prevotella multisaccharivorax and as-yet-uncultivated Bacteroidetes clone X083 in primary endodontic infections. J Endod. 2009;35(10):1359-62.

36. Siqueira JF Jr, Rocas IN. The microbiota of acute apical abscesses. J Dent Res. 2009;88(1):61-5.

37. Signori $\mathrm{CN}$, et al. Microbial diversity and community structure across environmental gradients in Bransfield Strait, Western Antarctic Peninsula. Front Microbiol. 2014;5:647.

\section{Figures}

a

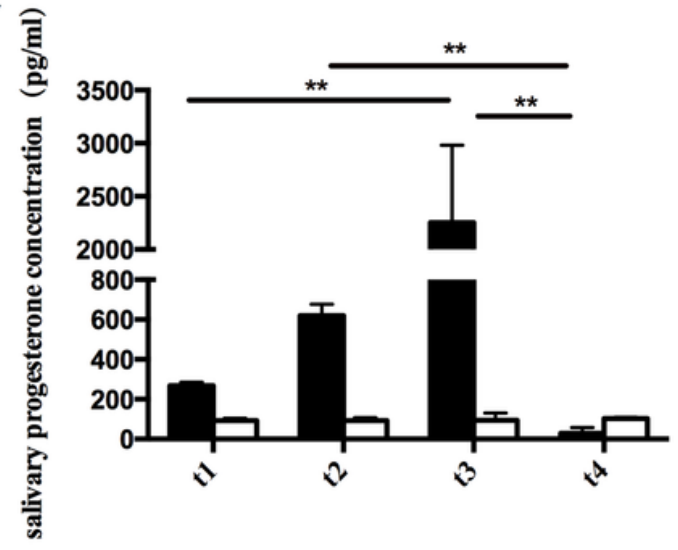

b

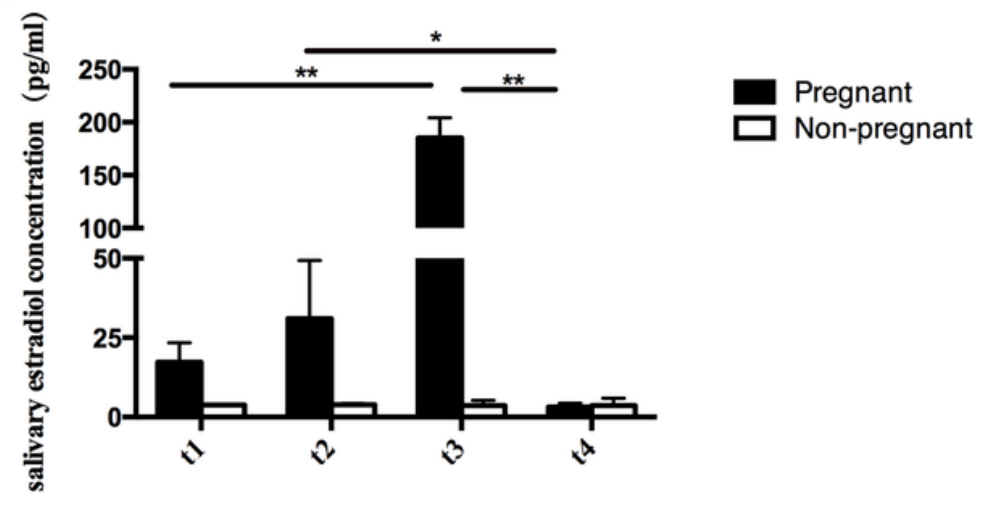

\section{Figure 1}

Concentration of salivary hormones during pregnancy and post-delivery. Presented as median (interquartile range). Salivary progesterone levels (a) and salivary estradiol levels (b). t1-t4 in the pregnant group represent the first trimester ( $\leq 14$ weeks), the second trimester (20-25 weeks), the third trimester (33-37 weeks) and postpartum (42 days after delivery), respectively, as well as a two-month interval in the non-pregnant group. The $y$-axis indicates the concentration $(\mathrm{pg} / \mathrm{ml})$. ${ }^{*} \mathrm{P}<0.01,{ }^{*} \mathrm{P}<0.05$. 
$\mathbf{a}$

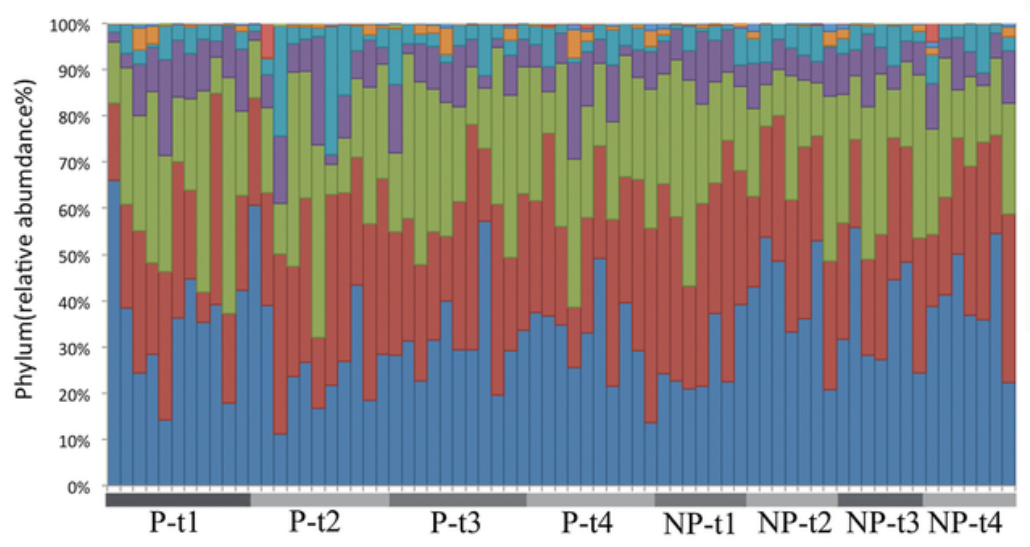

c

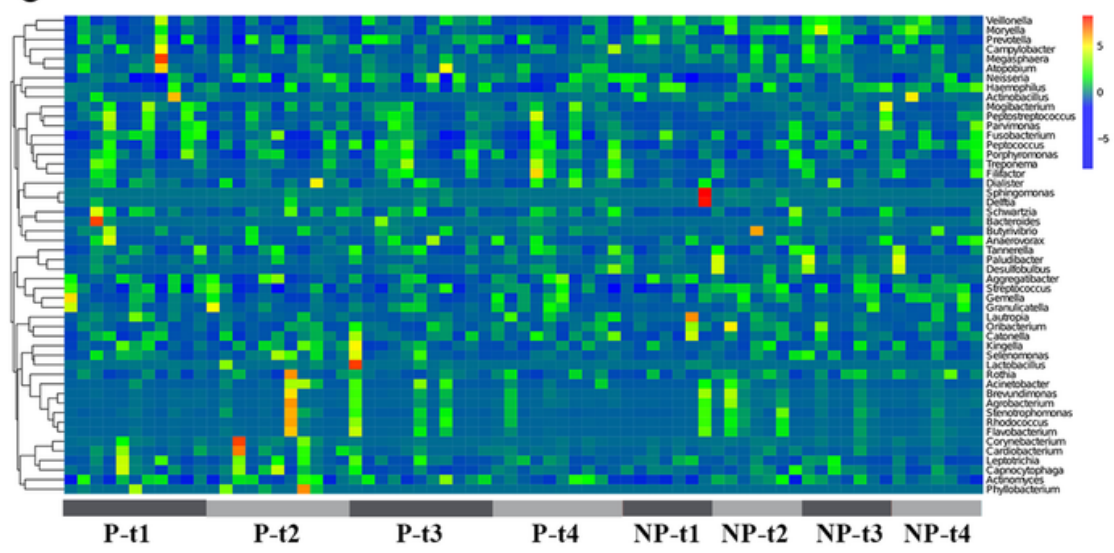

b

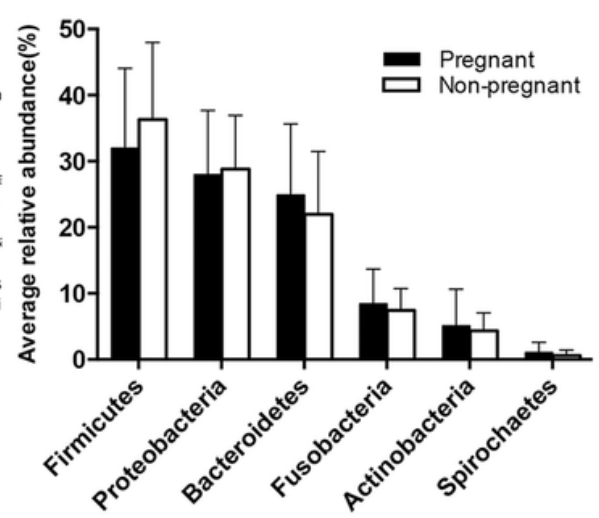

d

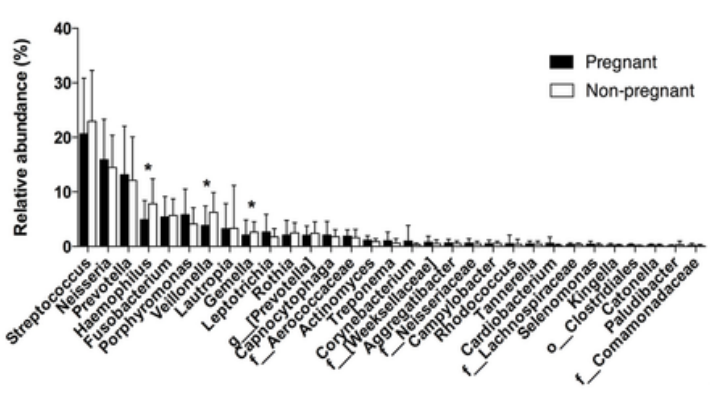

Figure 2

Overall composition of salivary microbiota in pregnant and non-pregnant groups. (a) The relative abundance of sixteen phyla in all samples. (b) Relative abundance of the phyla core compared in pregnant and non-pregnant groups. (c) Richness of the top 50 genera is shown in the heatmap. (d) The relative abundance of 31 genera core compared in pregnant and non-pregnant groups. ${ }^{*} P<0.05$. 


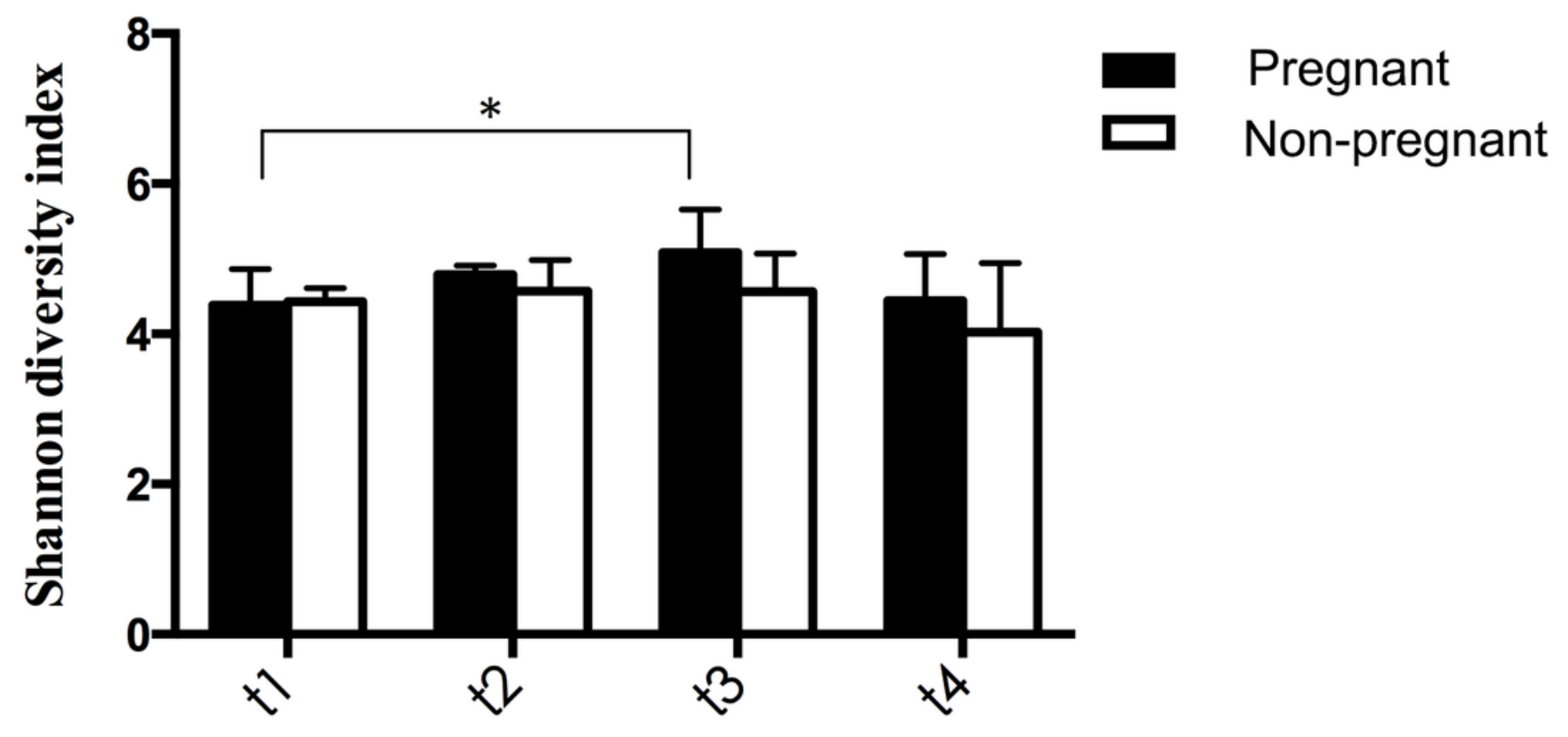

Figure 3

The diversity of the overall salivary microbiota calculated by the Shannon index. Presented as median (interquartile range). $\mathrm{t} 1: \leq 14$ weeks; $\mathrm{t} 2: 20-25$ weeks; $\mathrm{t} 3: 33-37$ weeks); $\mathrm{t} 4: 42$ days after delivery. * $\mathrm{P}<0.05$.

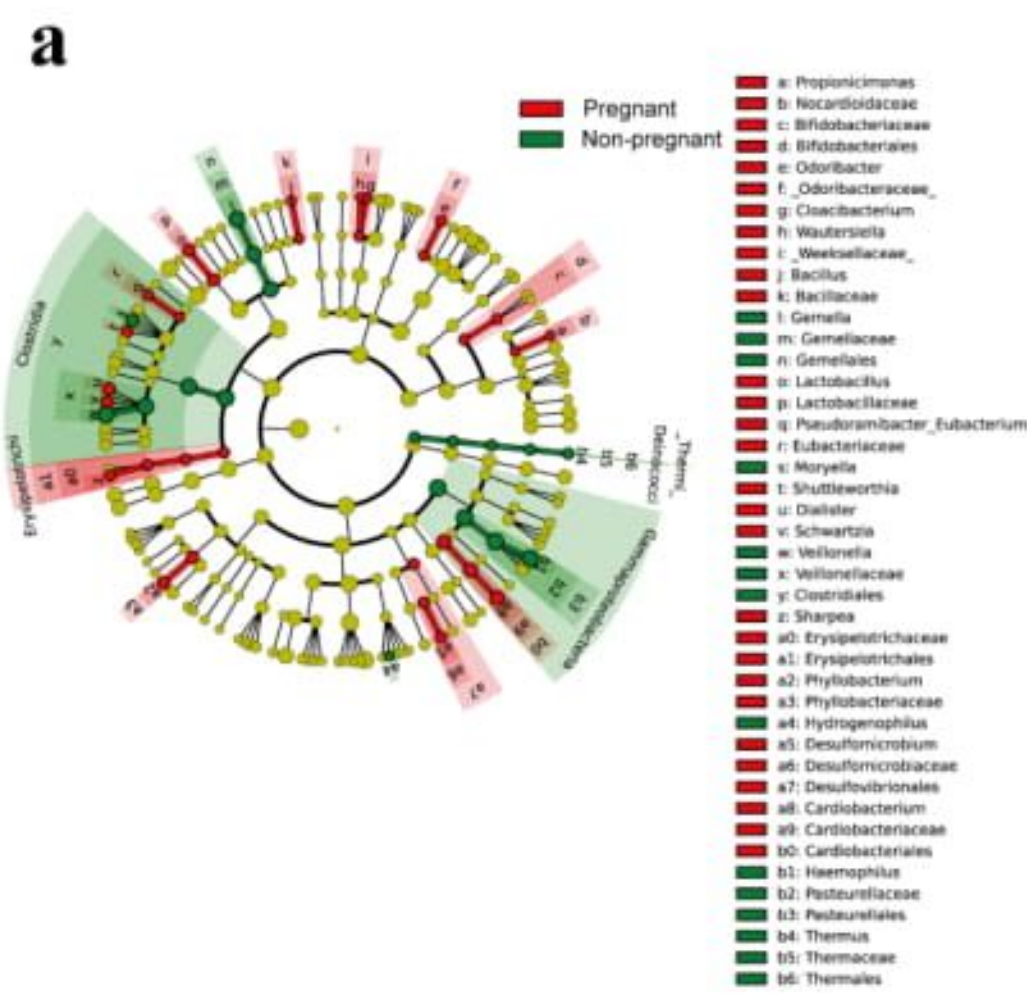

b

Figure 4 
(a) Differences of taxa between pregnant and non-pregnant women determined by linear discriminate analysis coupled with size effect (LEfSe) measurements. The color indicates the group in which differential taxa was most abundant. The red and green represent the pregnant and non-pregnant groups, respectively. The yellow indicates that there were no differences in this taxa between the two groups. (b) The LDA score of distinct genera.

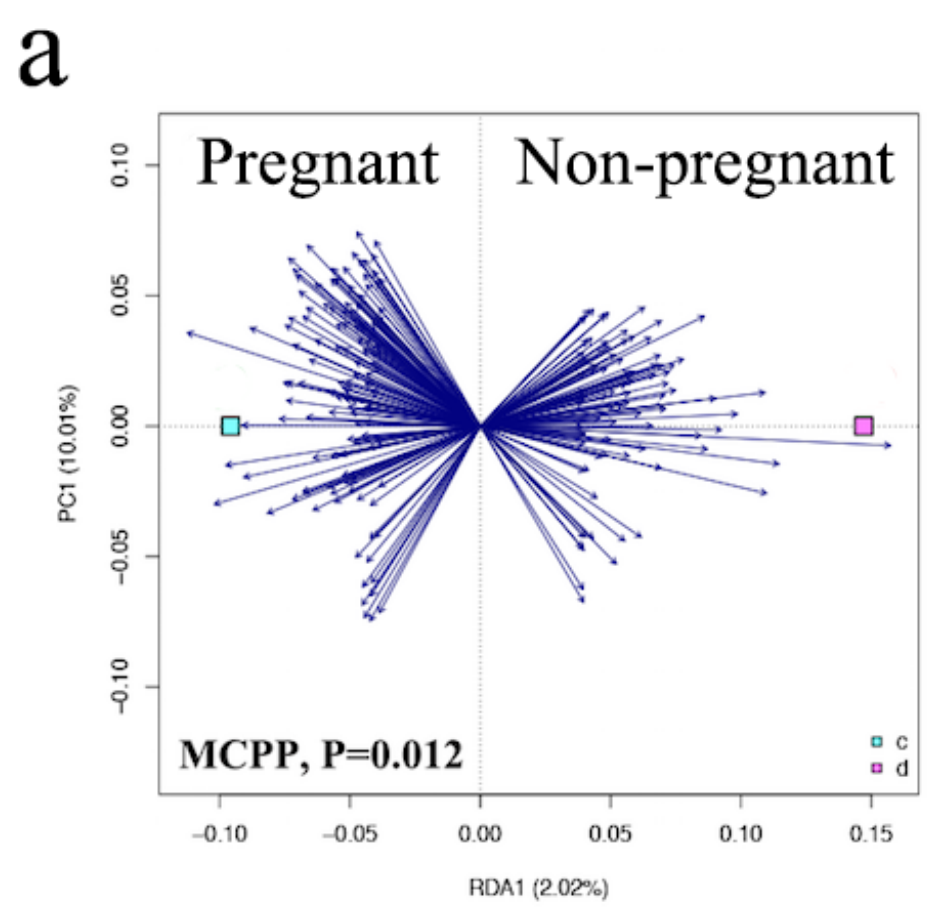

b
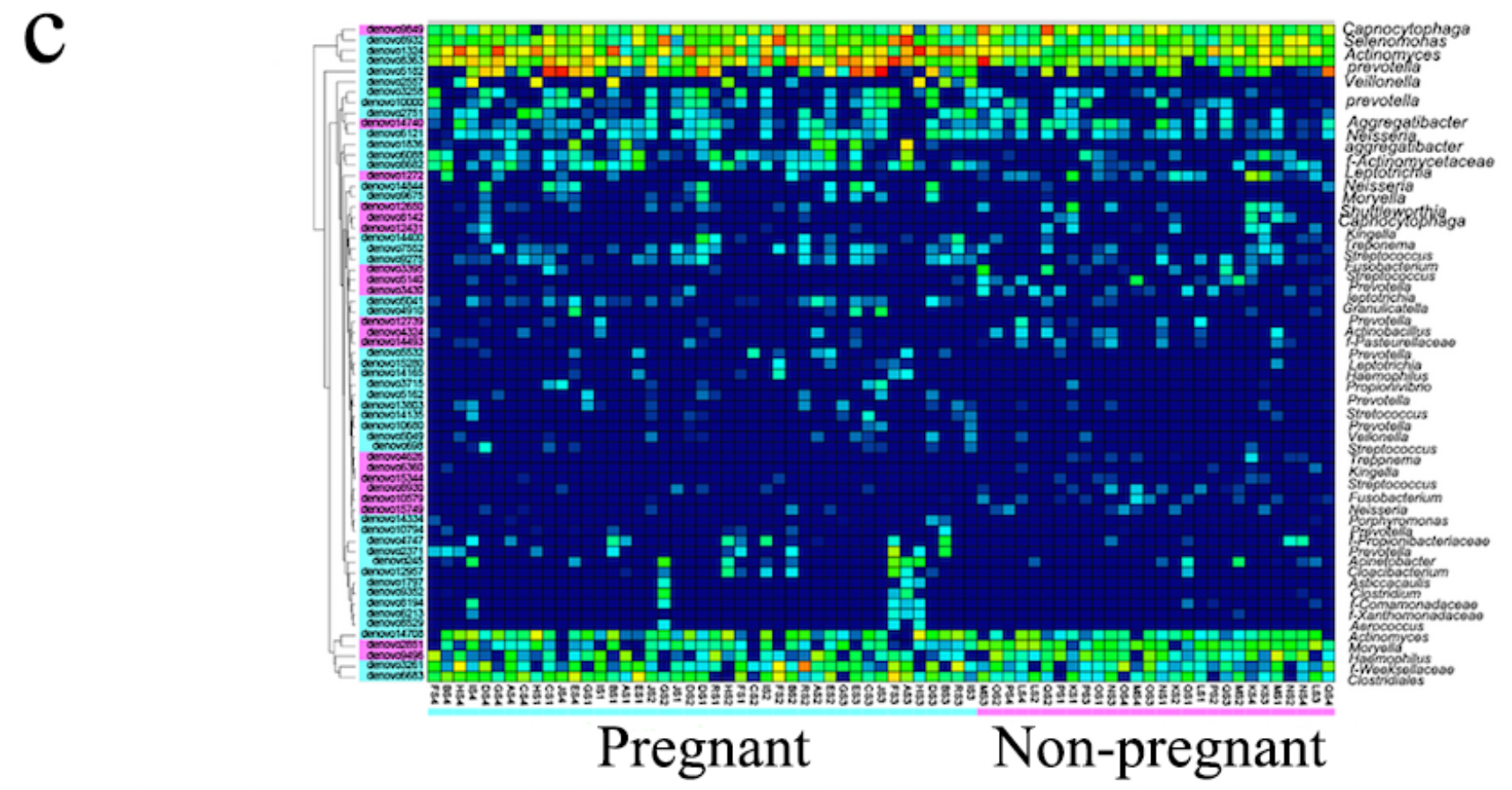

\section{Figure 5}

OTUs associated with salivary sex hormone levels identified by two-step RDA analysis. (a) Bioplot of the RDA of the salivary microbiota associated with pregnancy. Selected OTUs that have shared $2.02 \%$ variability of the salivary microbiota are indicated by blue arrows. (b) Bioplot of the RDA of the salivary 
microbiota related to sex hormone levels. Responding OTUs that have shared $1.68 \%$ of the variability of the oral microbiota are indicated by the blue arrows. (c) The relative abundances of the 63 OTUs identified by two steps of RDA. OTUs shown in green are more abundant in pregnant volunteers and those in red are more abundant in non-pregnant women.

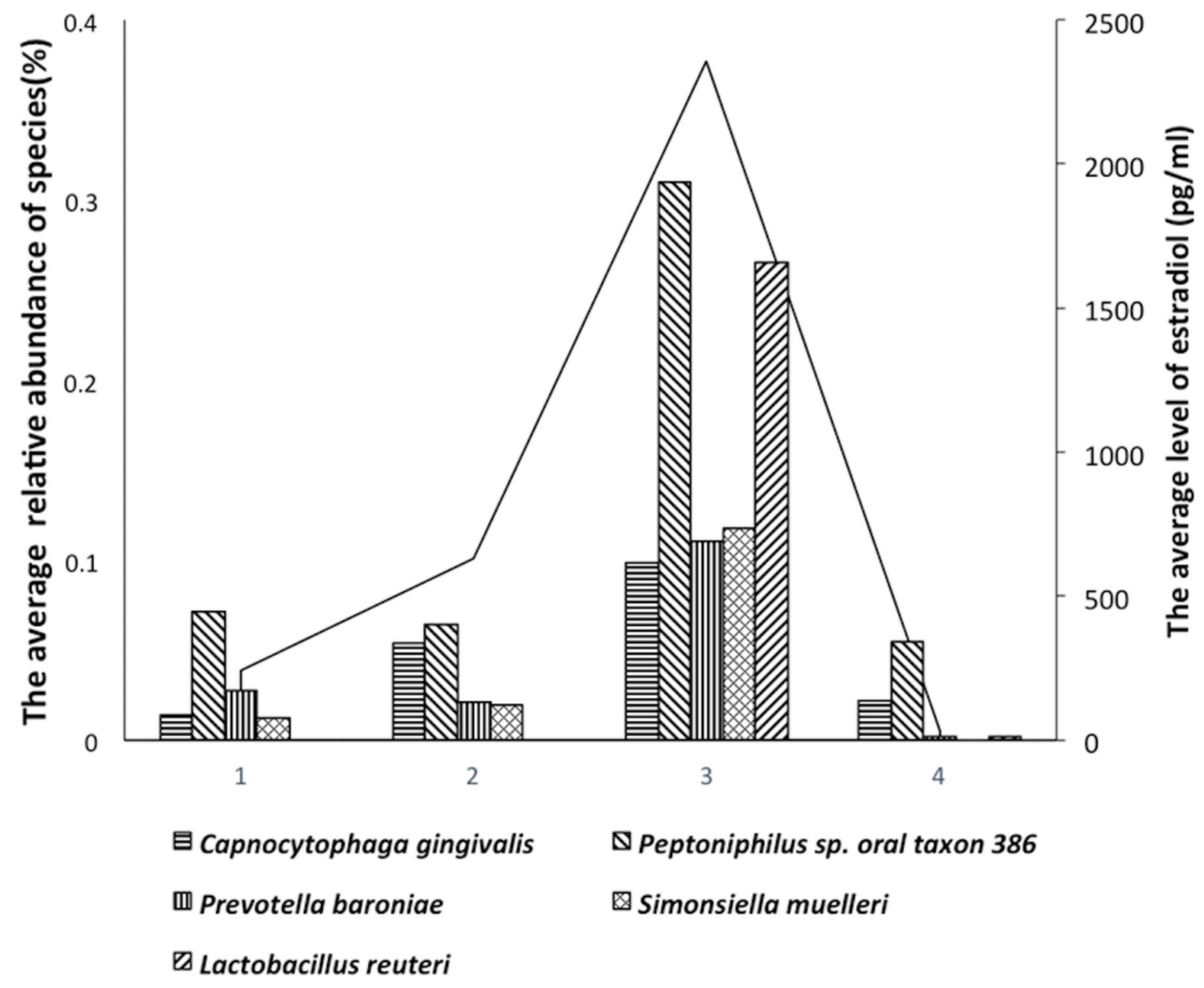

Figure 6

The correlation of species to salivary sex hormone levels by Pearson correlation test. The horizontal axis indicated $\mathrm{t} 1 \sim \mathrm{t} 4$ from left to right. The average estradiol levels during pregnancy and after delivery were presented as line chart.

\section{Supplementary Files}

This is a list of supplementary files associated with this preprint. Click to download. 
- Tables3.xls

- Tables2.docx

- Tables1.docx

- Figures2.tif

- Figures1.tif 